\title{
48. EXISTENCE OF METEOR SHOWERS ASSOCIATED WITH SHORT-PERIOD COMETS ANOMALOUS IN MOTION
}

\author{
Z. SEKANINA \\ (Centre for Numerical Mathematics, \\ Faculty of Mathematics and Physics, Charles University, Praha, Czechoslovakia)
}

\begin{abstract}
A BSTRACT
The observed properties of non-gravitational effects in the motion of the short-period comets are described in connection with the possible mass-loss processes in their nuclei. The impulses on the nuclei have been found to be about $1 \mathrm{~cm} / \mathrm{sec}$ to $3 \mathrm{~m} / \mathrm{sec}$ per revolution. The contributions to the observed dynamic anomalies of gas- and dust-matter ejections from the nucleus into the comet's atmosphere are estimated. The existence of meteor showers is related to the characteristics of cometary activity. Methods of further investigations are suggested.
\end{abstract}

\section{Non-Gravitational Phenomena}

At the present time there are no doubts about the real existence of non-gravitational phenomena in comet motions, and their detectability. Their effects on short-period comets may be summarized as follows:

(i) The most striking deviation from the purely gravitational law has always been ascertained in the daily mean motion, giving a rate of the comet's secular acceleration or deceleration.

(ii) The anomaly does not remain constant, but systematically decreases with time. It is likely that there exists a relation between the rate of acceleration and the comet's age.

(iii) There are suggestions (e.g. Backlund, 1911 and recently Makover, 1955 as regards Comet $\mathrm{P} /$ Encke) that the non-gravitational forces act more or less discontinuously near perihelion. However, it is possible that both abrupt and continuous components are present.

(iv) It is primarily the number of revolutions which determines the total change in the daily mean motion of a comet, not the time elapsed.

\section{Impulses on the Nucleus}

A concise list of dynamic impulses (in metres per second per revolution) exerted by non-gravitational forces on some comet nuclei is given in Table 1. A few additional data, preliminary in character, based on very recent computations by Marsden (1967), 
Table 1

Dynamic impulses on the nuclei of the short-period comets (per revolution)

\begin{tabular}{lccc}
\multicolumn{1}{c}{ Comet } & $w_{0}(\mathrm{~m} / \mathrm{sec})$ & Period of time & Motion \\
P/Encke & $2 \cdot 1-2 \cdot 9$ & $1786-1858$ & accelerated \\
& $1 \cdot 3-1 \cdot 8$ & $1858-1894$ & accelerated \\
& $0 \cdot 8-1 \cdot 0$ & $1894-1931$ & accelerated \\
P/Grigg-Skjellerup & $0 \cdot 3-0 \cdot 6$ & $1931-1957$ & accelerated \\
P/Pons-Winnecke & $\approx 0 \cdot 04$ & $1947-1961$ & accelerated \\
P/Giacobini-Zinner & $0 \cdot 02$ & $1858-1886$ & decelerated \\
P/Biela & $0 \cdot 5$ & $1933-1947$ & decelerated \\
P/d'Arrest & $1 \cdot 0$ & $1806-1832$ & accelerated \\
P/Brooks (2) & $0 \cdot 6-1 \cdot 0$ & $1851-1923$ & decelerated \\
& $0 \cdot 5$ & $1889-1910$ & accelerated \\
P/Wolf & $0 \cdot 3-0 \cdot 4$ & $1925-1953$ & accelerated \\
& $0 \cdot 02-0 \cdot 03$ & $1884-1918$ & decelerated \\
& $0 \cdot 006$ & $1925-1958$ & decelerated
\end{tabular}

are included in Table 2. The observed impulses can be shown to represent a rather high momentum of the matter responsible.

Let us estimate the contribution of the gas and solid-matter action to the total dynamic effect observed on $\mathrm{P} /$ Encke, for example. The total number of molecules emitted is certainly less than $10^{31}$, or some $5 \times 10^{8}$ grammes per revolution. The probability of the emission of gases is estimated at some 0.1 at a unit heliocentric distance, and the velocity of ejection is hardly in excess of $1 \mathrm{~km} / \mathrm{sec}$. Thus the total 'unfocussed' molecular impulse on the nucleus of $\mathbf{P} /$ Encke is certainly less than $10^{15}$ cgs. The probable value is some 1-2 orders lower, and the effect of the 'focussed' flow one more order lower. But Tables 1 and 2 show that the nucleus is exposed to impulses of $10^{18} \mathrm{cgs}$ per revolution, if a mass as low as $10^{16}$ grammes is adopted for the nucleus. A discrepancy of at least three orders, but most probably some 5-7 orders, results. To remove it, we should have to assume that we are able to detect only each millionth molecule actually present in the comet's head. The same is true for

Table 2

Impulses from the $\Delta T$ deviations by Marsden (Preliminary values)

\begin{tabular}{lccc}
\multicolumn{1}{c}{ Comet } & $w_{0}(\mathrm{~m} / \mathrm{sec})$ & Period of time & Motion \\
P/Encke & $(0 \cdot 6)$ & $1947-1964$ & accelerated \\
P/Honda-Mrkos- & 2 & $1948-1964$ & accelerated \\
$\quad$ Pajduśáková & $\approx 0$ & $1939-1964$ & - \\
P/Pons-Winnecke & 0.2 & $1942-1961$ & decelerated \\
P/Forbes & 0.7 & $1943-1963$ & decelerated \\
P/d'Arrest & $>0$ & $1933-1964$ & $?$ \\
P/Whipple & & &
\end{tabular}




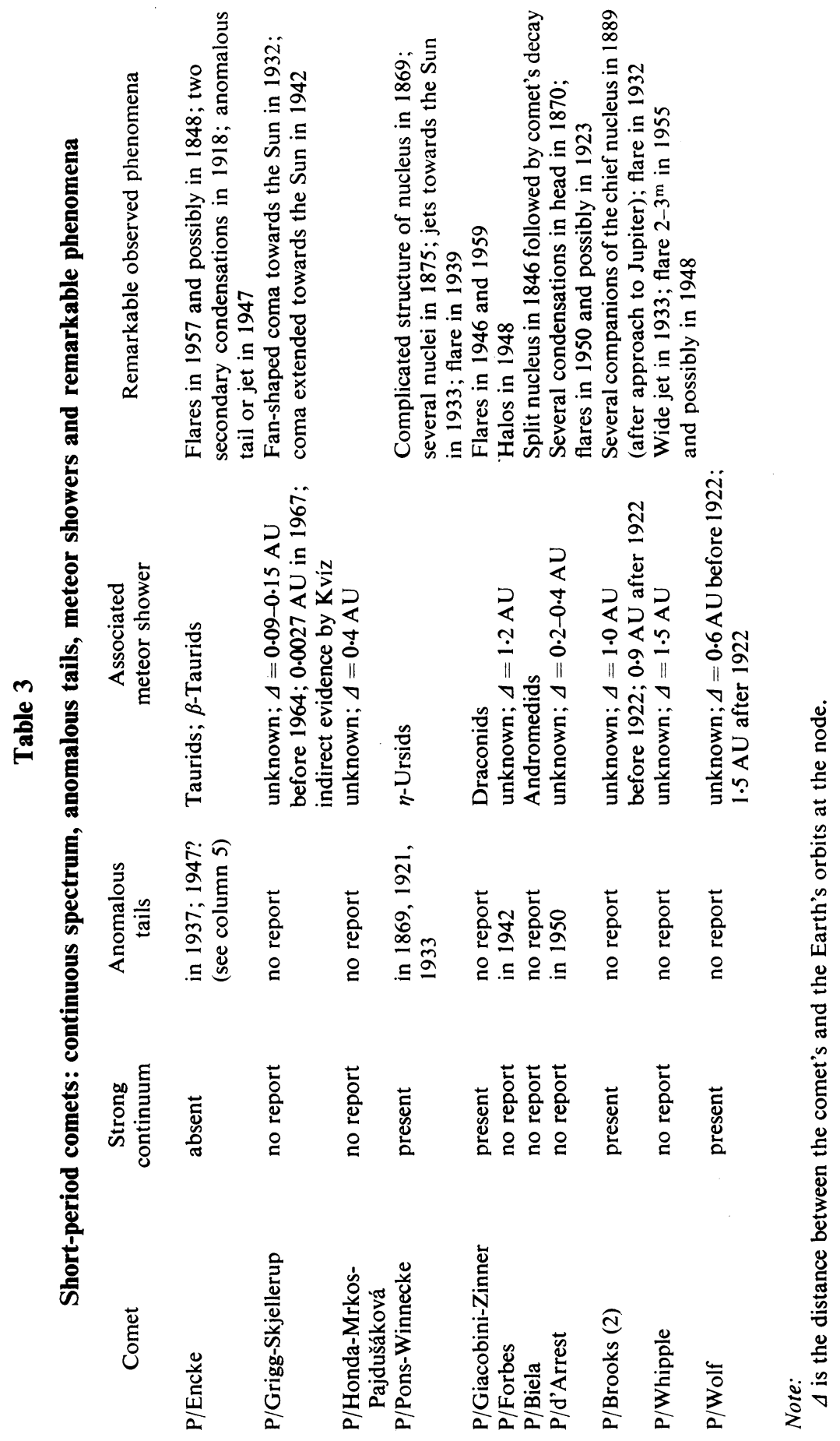


any other short-period comet of Tables 1 and 2. This estimate argues against the concept that the gas by itself could be responsible.

In view of this, the data on the existence of the continuous spectrum, anomalous tail and a meteor stream associated with the comet, as well as on various remarkable phenomena observed in the head, are of high interest for testing whether the dust particles stand a good chance of explaining the dynamic deviations. For the eleven comets, these data are summarized in Table 3, compiled from various sources. As is seen, non-stationary processes were observed in practically all the comets in question. An associated meteor shower was reported for each of the comets (approaching close enough to the Earth's orbit), except P/Grigg-Skjellerup. The orbit of this comet had missed the Earth's orbit at some 0.09 to 0.15 AU before the comet's approach to Jupiter in 1964. The strong perturbations by the planet during the approach made the conditions for the detection of the possible shower - the Puppids - much more favourable since 1967 (Sitarski, 1964). In addition, there exists indirect evidence on the probable existence of a cloud of solid particles associated with this comet from the investigation of precipitation singularities by Kvíz (1965).

At least for $\mathrm{P} /$ Encke no continuum has ever been observed though there exists an associated meteor stream. But there are no spectroscopic observations of the anomalous tails of this comet, which should give a continuum. Moreover, the Taurids are a typical old shower, several thousand years in age, while the spectroscopic observations of $\mathrm{P} /$ Encke date since its 1871 apparition only. However, the non-gravitational forces act even in the period of the 'continuumless' comet, which leads to either of the two conclusions:

(i) Separations of the meteoric matter from the nucleus are not responsible for the observed dynamic impulses on the latter.

(ii) The impulses are exerted by discrete ejections of the meteoric matter (anomalous tails), having thus expresly an abrupt character.

Energy considerations, analogous to those above regarding the gas, speak in favour of the former of the two conclusions, but not quite decisively.

Table 3 indicates that the nuclei of the investigated short-period comets are rather unstable, inclining to various explosive phenomena (flares, splits, etc.). Even the nucleus of $\mathrm{P} /$ Encke, a typical inactive comet, tends to such processes. A number of them, taking place in the nuclear region of the head, need not be, moreover, visible to terrestrial observers. Especially the phenomena of fragmentation of the comet's nucleus by splitting off large enough blocks of solid matter (without sufficient supplies of gases to form atmospheres of their own) would be ineffective photometrically, but decisive dynamically.

To gather more information on the character of the non-gravitational forces in the short-period comets, and on their connection with the ejection of solid matter from the comet nuclei, the following investigations would be most useful: 
(1) An analysis of the structure of nuclei of the short-period comets by means of space missions.

(2) A study of the occurrence of anomalous tails in these comets and their careful photometric and spectroscopic observations.

(3) A study of the occurrence of bright bolides connected with the meteor showers, to detect larger blocks of the solid matter thrown out from the comet nuclei.

\section{References}

Backlund, O. (1911) Mem. Acad. Imp. Sci., St. Pétersbourg, Sér. 8, 30, No. 2.

Kvíz, Z. (1965) Meteorologické zprávy, 18, 177.

Makover, S.G. (1955) Trudy Inst. teor. Astr., 4, 133.

Marsden, B.G. (1967) Report to Comm. 20, 13th IAU General Assembly, Prague.

Sitarski, G. (1964) Acta astr., 14, 25.

\section{DISCUSSION}

Lancaster Brown: What is your definition of anomalous tails as used in your paper?

Sekanina: The anomalous tail was understood to be any ejection out of the boundaries of the visible coma in a direction more or less opposite to the prolonged radius-vector. 